\title{
A Study on the Effects of Data Accuracy and Datum Inconsistencies on Relative GPS Levelling
}

\author{
C. Kotsakis, G. Fotopoulos and M.G. Sideris \\ Department of Geomatics Engineering, University of Calgary \\ 2500 University Drive N.W., Calgary, Alberta, Canada, T2N 1N4, Email: gfotopou@ucalgary.ca
}

\begin{abstract}
Numerical investigations into the effects of data accuracy and datum inconsistencies on relative GPS levelling are presented. Specifically, the variance/covariance information of (i) relative GPS ellipsoidal heights, (ii) geoid heights computed from a gravimetric geoid model, and (iii) orthometric heights obtained from spirit levelling methods, is used for an accuracy analysis in a combined 1D multi-data test network of GPS levelling benchmarks. Different parametric models are employed to describe the datum inconsistencies and systematic distortions inherent among the various height data sets. The a-posteriori accuracy of the adjusted parameters in the corrector surface model, along with the internal accuracy of the GPS and geoid heights, are finally used to infer the achievable accuracy of GPS levelling on baselines within the test network area.
\end{abstract}

\section{Introduction}

The optimal combination of geometric heights obtained from global positioning system (GPS) measurements and geoidal undulations computed from a gravimetric geoid model, in order to determine orthometric heights with respect to a vertical geodetic datum, is well suited for many practical applications. This process, referred to as GPS levelling, is based on a simple geometrical relationship that binds the three height types and is given by (Heiskanen and Moritz, 1967):

$$
H=h-N
$$

where, $h$ is the geodetic/ellipsoidal height, $H$ refers to the Helmert orthometric height, and $N$ is the geoidal undulation obtained from a gravimetric geoid model. In practice, the relationship given by Eq. (1) is never fulfilled due to numerous errors, systematic distortions and datum inconsistencies inherent among the triplet of height data (Kotsakis and Sideris, 1999). Thus, a more rigorous treatment for the integration of these different height types requires the incorporation of a parametric corrector surface model in Eq. (1). The role of such a model is to absorb the datum inconsistencies and any systematic distortions that exist in the height data sets.

The purpose of this paper is to analyze the impact of the GPS and geoid data accuracy, and the accuracy of the corrector surface model parameters, on the determination of relative orthometric heights (relative GPS levelling). A test network of spirit levelled GPS control points situated in the western part of Canada is used as a basis for all numerical investigations. Using this network, various simulative adjustments are performed in order to determine the covariance $(\mathrm{CV})$ matrix for the estimated parameters in the corrector surface model. By using this accuracy information, evaluations on the achievable accuracy of GPS-derived orthometric heights for new baselines (not included in the original network adjustment) is performed. A number of different scenarios are studied by varying the configuration of the test network and the location/length of the new baseline.

\section{General Methodology}

Given a network of points with known GPS and orthometric height values and the availability of a gravimetric geoid model, a combined adjustment of GPS/levelling/geoid data can be performed. In this paper, the height differences for each data type are formed with respect to some selected initial point/station. Therefore, the "observed" input values are $\Delta h_{i j}, \Delta H_{i j}$, and $\Delta N_{i j}$ for a pair of points $(i, j)$ forming a baseline in the test network. The corresponding observation equation model is given by:

$\Delta h_{i j}=h_{j}^{\alpha}-h_{i}^{\alpha}+f_{i j}^{\Delta h}+v_{i j}^{\Delta h}$ 


$$
\begin{aligned}
& \Delta H_{i j}=H_{j}^{\alpha}-H_{i}^{\alpha}+f_{i j}^{\Delta H}+v_{i j}^{\Delta H} \\
& \Delta N_{i j}=N_{j}^{\alpha}-N_{i}^{\alpha}+f_{i j}^{\Delta N}+v_{i j}^{\Delta N}
\end{aligned}
$$

where the superscript $\alpha$ denotes the true values of the various heights at each point, and $f_{i j}^{(\cdot)}$ describes the systematic errors and datum inconsistencies in the height data sets. The true values $h_{i}^{\alpha}, H_{i}^{\alpha}, N_{i}^{\alpha}, h_{j}^{\alpha}, H_{j}^{\alpha}$, and $N_{j}^{\alpha}$ refer to a common datum such that the following conditions are satisfied:

$$
\begin{aligned}
& h_{i}^{\alpha}-H_{i}^{\alpha}-N_{i}^{\alpha}=0 \\
& h_{j}^{\alpha}-H_{j}^{\alpha}-N_{j}^{\alpha}=0 \\
& \Delta h_{i j}^{\alpha}-\Delta H_{i j}^{\alpha}-\Delta N_{i j}^{\alpha}=0
\end{aligned}
$$

and $v_{i j}^{(\cdot)}$ denotes the zero mean random errors for each height difference. The final synthetic observation equation for each baseline in the test network will have the following form:

$$
\ell_{i j}=f_{i j}+v_{i j}^{\Delta h}-v_{i j}^{\Delta H}-v_{i j}^{\Delta N}
$$

where $\ell_{i j}=\Delta h_{i j}-\Delta H_{i j}-\Delta N_{i j}$ is the 'observed' misclosure value for each baseline, and $f_{i j}$ refers to the total (combined) correction term for systematic errors and datum inconsistencies in the multi-data test network.

The $f_{i j}$ term in Eq. (4) can be modelled according to the following deterministic parametric form:

$$
f_{i j}=\left(\mathbf{a}_{j}^{T}-\mathbf{a}_{i}^{T}\right) \mathbf{x}=\mathbf{a}_{i j}^{T} \mathbf{x}
$$

where $\mathbf{a}_{j}, \mathbf{a}_{i}$ are $n \times 1$ vectors of known coefficients that depend on the horizontal location of the network points $i, j$ and $\mathbf{x}$ is an $n \times 1$ vector of unknown parameters. An example of such a parametric model for the relative case is given by:

$$
\mathbf{a}_{i j}=\left[\begin{array}{c}
\cos \varphi_{j} \cos \lambda_{j}-\cos \varphi_{i} \cos \lambda_{i} \\
\cos \varphi_{j} \sin \lambda_{j}-\cos \varphi_{i} \sin \lambda_{i} \\
\sin \varphi_{j}-\sin \varphi_{i}
\end{array}\right] \mathbf{x}=\left[\begin{array}{c}
x_{1} \\
x_{2} \\
x_{3}
\end{array}\right]
$$

where $\varphi$ and $\lambda$ are the horizontal geodetic coordinates, and $x_{1}, x_{2}$ and $x_{3}$ are the unknown parameters. This formulation follows the classical pointwise (absolute) four-parameter transformation model given in Heiskanen and Moritz (1967, sec. 59), with the omission of the fourth constant parameter which cancels out in the relative case.

The choice of the parametric form of the corrector surface model is not a trivial task. Candidate models vary from a simple plane to more complicated higher-order polynomial and/or trigonometric corrector surfaces (De Bruijne et al., 1997; Featherstone, 2000). For the tests conducted in this paper, three different parametric models were examined for the relative case, namely Eq. (6) and two additional models as given below:

$\mathbf{a}_{i j}=\left[\begin{array}{c}\cos \varphi_{j} \cos \lambda_{j}-\cos \varphi_{i} \cos \lambda_{i} \\ \cos \varphi_{j} \sin \lambda_{j}-\cos \varphi_{i} \sin \lambda_{i} \\ \sin \varphi_{j}-\sin \varphi_{i} \\ \sin ^{2} \varphi_{j}-\sin ^{2} \varphi_{i}\end{array}\right] \mathbf{x}=\left[\begin{array}{c}x_{1} \\ x_{2} \\ x_{3} \\ x_{4}\end{array}\right]$

and

$$
\begin{aligned}
& \mathbf{a}_{i j}=\left[\begin{array}{lllllll}
\delta \lambda_{i j} & \delta \varphi_{i j} & \delta \lambda_{i j} \delta \varphi_{i j} & \delta \varphi_{i j}^{2} & \delta \lambda_{i j}^{2} & \delta \varphi_{i j}^{3} & \delta \lambda_{i j}^{3}
\end{array}\right]^{T} \\
& \mathbf{x}=\left[\begin{array}{lllllll}
x_{1} & x_{2} & x_{3} & x_{4} & x_{5} & x_{6} & x_{7}
\end{array}\right]^{T}
\end{aligned}
$$

The model in Eq. (7) is a simple extension of the model in Eq. (6), see Heiskanen and Moritz (1967, p.207). The model given in Eq. (8) is a sevenparameter third-order polynomial composed of nontrigonometric basis functions. Similar choices have been made in other studies (see, e.g., Jiang and Duquenne, 1995)

The $v_{i j}^{(\cdot)}$ terms in Eq. (4) describe the zero mean random errors in the GPS, levelling and geoid height differences. Their second-order stochastic properties, for all baselines in the multi-data network, are provided by the following covariance matrices:

$$
\begin{aligned}
& E\left\{v_{\Delta h} v_{\Delta h}^{T}\right\}=C_{\Delta h}, E\left\{v_{\Delta H} v_{\Delta H}^{T}\right\}=C_{\Delta H}, \\
& E\left\{v_{\Delta N} v_{\Delta N}^{T}\right\}=C_{\Delta N}
\end{aligned}
$$

where $E$ denotes the mathematical expectation operator. The evaluation of these $\mathrm{CV}$ matrices will be discussed in more detail in the following section. 
Given the theoretical relationship among the three types of height data, and the incorporation of an appropriate parametric corrector surface model, the orthometric height difference for a new (i.e. not belonging in the original test network) baseline $\mathrm{kl}$ as obtained from relative GPS levelling is:

$$
\Delta H_{k l}=\Delta h_{k l}-\Delta N_{k l}-\left(\mathbf{a}_{l}^{T}-\mathbf{a}_{k}^{T}\right) \hat{\mathbf{x}}
$$

where $\hat{\mathbf{x}}$ is the vector containing the estimated parameters of the corrector surface model, as obtained from the adjustment of the synthetic observations (Eq. 4) in the test network (for more details, see Kotsakis and Sideris, 1999). For our study, we are interested in the achievable accuracy for the orthometric height difference $\Delta H_{k l}$. This accuracy can be evaluated by simply applying variance-covariance propagation to Eq. (10), resulting in the following formula:

$$
\sigma_{\Delta H_{k l}}^{2}=\sigma_{\Delta h_{k l}}^{2}+\sigma_{\Delta N_{k l}}^{2}+\left(\mathbf{a}_{l}^{T}-\mathbf{a}_{k}^{T}\right) C_{\hat{x}}\left(\mathbf{a}_{l}^{T}-\mathbf{a}_{k}^{T}\right)^{T}
$$

where $\sigma_{\Delta h_{k l}}^{2}$ and $\sigma_{\Delta N_{k l}}^{2}$ are the variances of the relative ellipsoidal and geoidal heights of the new baseline, and $C_{\hat{x}}$ is the a-posteriori $\mathrm{CV}$ matrix of the adjusted parameters in the corrector surface model. This CV matrix is obtained from the multidata adjustment of the GPS/levelling/geoid test network. Due to space restrictions, more details and mathematical formulas for the derivation of the $C_{\hat{x}}$ matrix will be provided in a future journal paper by the authors.

\section{Test Description}

Although this study does not make use of actual height data values, the height accuracy information that is used and the test network configuration are simulated to mirror realistic conditions. The selected test network consists of a subset of the GPS benchmarks in the south-western part of Canada (314 points in Alberta and British Columbia) covering $49^{\circ} \leq \varphi \leq 55^{\circ}$ and $-120^{\circ} \leq \lambda \leq-110^{\circ}$, which translates to an approximate network coverage area of $667 \mathrm{~km} \times 685 \mathrm{~km}$ as depicted in Figure 1.

Full CV matrices for the GPS and orthometric heights at the test network points were obtained through separate simulative adjustments, which used the measuring accuracy of GPS and spirit levelling as input, according to the following standard formulation:

$\sigma(m m)=b \cdot \sqrt{d}$

where $\sigma$ is the standard deviation of the observed height differences in $\mathrm{mm}, b$ is the height difference accuracy based on published standards for GPS and levelling, and $d$ is the baseline length in $\mathrm{km}$ (Kearsley et al., 1993).

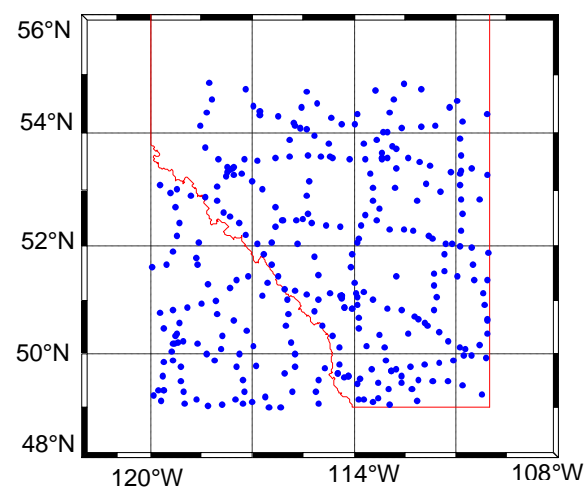

Fig. 1 Distribution of test network points

Three different orders of levelling accuracy were used for assigning the a-priori value $\sigma_{\Delta H}$ for each baseline in the adjustment of the levelling network (Fig. 2), namely $0.7 m m \sqrt{d}, 1.3 m m \sqrt{d}$, and $2 m m \sqrt{d}$, referring to first, second and third order respectively. National standards for the accuracy of vertical control vary depending on the country (Kearsley et al., 1993; Ollikainen, 1997; van Onselen, 1997). In our case, the U.S. standards were implemented for both levelling and GPS, as they were readily available (NGS, 1994). For the case of levelling, larger baselines $(d>80 \mathrm{~km})$ usually constitute part of a national levelling campaign and adhere to first order levelling standards, followed by denser regional levelling campaigns $(30 \mathrm{~km}<d \leq 80 \mathrm{~km}$ ) of second order, and finally local levelling lines $(d \leq 30 \mathrm{~km})$ which are of third order accuracy.

The accuracy for observed GPS height differences also degrades as the baseline length increases, mainly due to the spatial decorrelation of atmospheric GPS errors (Fotopoulos, 2000). In our analysis, ten different orders for the ellipsoidal 
height accuracy are used as they are defined by the U.S. standards (NGS, 1994). The a-priori accuracy $\sigma_{\Delta h}$ for each baseline in the separate adjustment of the GPS network was assigned based on the baseline length. For every $10 \mathrm{~km}$ increase in baseline length the value of $\sigma_{\Delta h}=b \sqrt{d}$ changed according to $b=(0.5,0.7,1,1.3,2,3,6,15,30,60)$ $\mathrm{mm}$ (e.g. for $d=55 \mathrm{~km}, b=3 \mathrm{~mm}$ ). The GPS network geometry used for the adjustment is shown in Fig. 2 (bottom). It is evident by comparing the two network configurations in Fig. 2 that the levelling network geometry is weaker than the GPS network due to the stringent line-of-sight restrictions of spirit levelling (Ollikainen 1997).
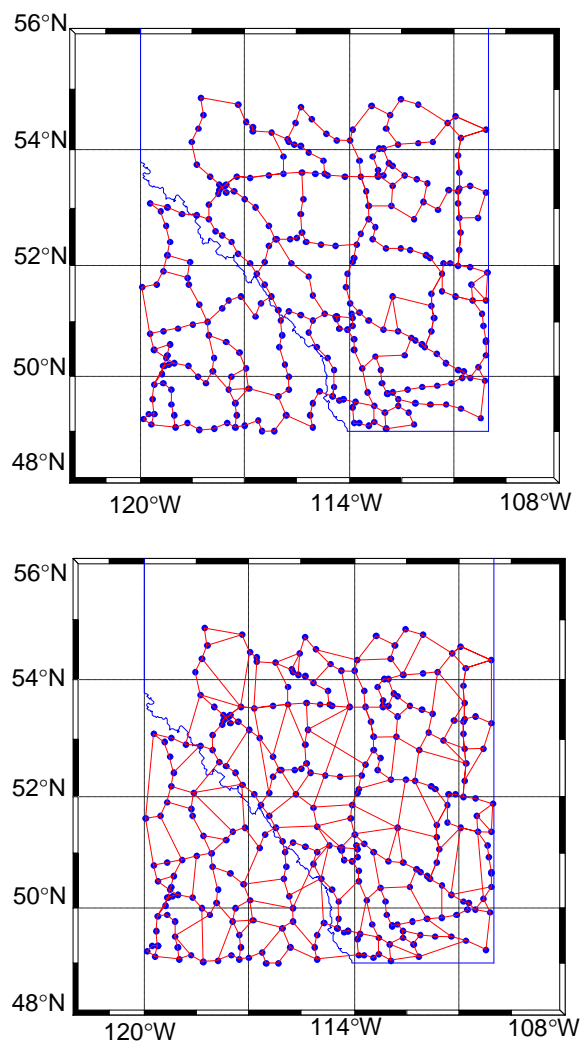

Fig. 2 Levelling (top) and GPS (bottom) Network Configurations

Following the separate adjustments of the levelling and GPS networks, full $\mathrm{CV}$ matrices, $C_{\Delta H}$ and $C_{\Delta h}$, for the adjusted height differences were obtained. These CV matrices were used as input to a final integrated multi-data adjustment in order to obtain $C_{\hat{x}}$ (see Eq. 11). The input accuracy for the geoid undulation differences in the multi-data test network was approximated by a diagonal $\mathrm{CV}$ matrix $C_{\Delta N}$. Its diagonal values $\sigma_{\Delta N}^{2}$ were based on the performance evaluation of the GSD95 Canadian geoid model in Alberta and British Columbia (Véronneau, 1997). Specifically, baselines in the flat or rolling hills area of the network were assigned a relative geoid accuracy of $1 \mathrm{~cm}+4 \mathrm{~mm} / \mathrm{km}$, and those in more mountainous regions were assigned a lower accuracy of $1 \mathrm{~cm}+10 \mathrm{~mm} / \mathrm{km}$. Therefore the relative geoid accuracy depended both on the spatial separation of the points, as well as on the geographical location of the baseline.

\section{Analysis of Results}

A summary of the various test network configurations that were used is provided in Table 1. The three network configurations labeled dense, mixed and sparse refer to the multi-data (GPS/levelling/geoid) test networks used to compute the a-posteriori accuracy $C_{\hat{x}}$ of the corrector surface model parameters. The different configurations for the multi-data test networks were selected in order to assess the effect of varying network geometry on the accuracy of relative GPS levelling for a new baseline that was not included in the original test network (see Fig. 3).

Table 1. Test Network Configuration Profiles ( $d$ denotes the baseline length, in $\mathrm{km}$ )

\begin{tabular}{l|cccc}
\multicolumn{5}{c}{$(d$ denotes the baseline length, in km) } \\
\hline Network & \# obs & $\mu(d)$ & $\sigma(d)$ & Max $(d)$ \\
\hline Levelling & 390 & 27.9 & 13.5 & 95.2 \\
GPS & 472 & 36.9 & 25.4 & 164.3 \\
Dense & 514 & 41.1 & 33.3 & 438.2 \\
Mixed & 425 & 32.3 & 20.3 & 128.1 \\
Sparse & 408 & 30.6 & 18.9 & 128.1 \\
\hline
\end{tabular}

In Table 2, the results of the achievable accuracy for relative GPS levelling, according to the procedure described in the previous sections (see Eq. 11) are shown for baselines of varying lengths, from a minimum of $10 \mathrm{~km}$ to a maximum of $100 \mathrm{~km}$. These results correspond to the averaged multi-data accuracy levels for baselines tested within and on the edge of the borders of the test networks (dense, mixed and sparse). The type of parametric model used was also varied according to Eqs. (6), (7) and (8). By examining the values in Table 2 , it became evident that the input accuracy of the geoidal height differences $\Delta N_{k l}$ and the GPS height differences 
$\Delta h_{k l}$ overshadowed any contribution from the parametric surface model, surpassing the contribution of this third term in Eq. (11) by several orders of magnitude. This is also the reason for not distinguishing in Table 2 between results from the different network configurations and parametric model types used, as the results did not vary significantly.

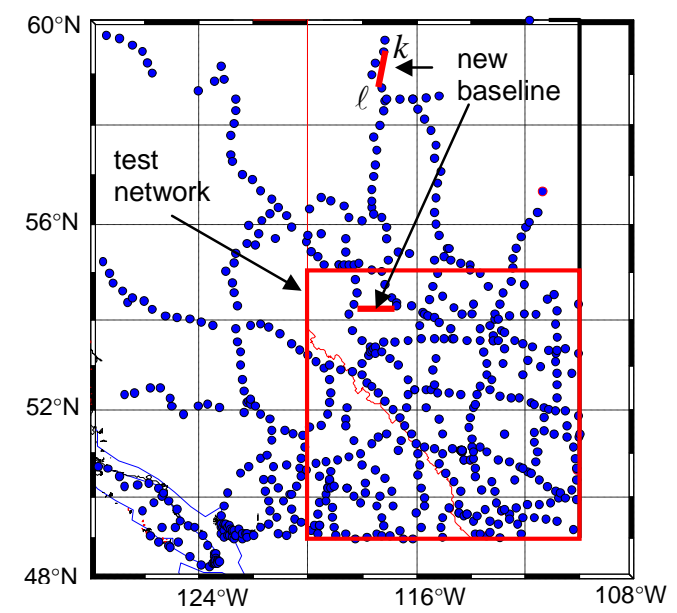

Fig. 3 New baselines within and outside the test network

Table 2. Standard error of relative height components (cm)

\begin{tabular}{c|ccc}
\hline$d(\mathrm{~km})$ & $\sigma_{\Delta h_{k l}}$ & $\sigma_{\Delta N_{k l}}$ & $\begin{array}{c}\sigma_{\Delta H_{k l}} \\
\text { from Eq. }(11)\end{array}$ \\
\hline 10 & 4.74 & 5.00 & 6.89 \\
20 & 6.71 & 9.00 & 11.23 \\
30 & 8.22 & 13.00 & 15.38 \\
40 & 9.49 & 17.00 & 19.47 \\
50 & 10.61 & 21.00 & 23.53 \\
60 & 11.62 & 25.00 & 27.57 \\
70 & 12.55 & 29.00 & 31.60 \\
80 & 13.42 & 33.00 & 35.62 \\
90 & 14.23 & 37.00 & 39.64 \\
100 & 15.00 & 41.00 & 43.66 \\
\hline
\end{tabular}

Since the internal accuracy of the GPS and geoid heights is independent of the type of the parametric corrector surface used and the network configuration, it is more illuminating to base the discussion of the results on the contribution of the third term in Eq. (11). This third term contains the contribution of the adjusted parameters of a prespecified model to the final GPS levelling accuracy, and is plotted for a number of different cases in Fig. 4. In this figure, each error profile/line is labeled based on an abbreviation which states the test network configuration ('d', 's', 'm' for dense, sparse and mixed respectively), followed by a 3 or 7 for the model type as shown in Eqs. (6) and (8), respectively. The results for the 4-parameter model were also computed, however they were very similar to the 3-parameter model values and therefore omitted to avoid cluttering the graph.

The results in Fig. 4 depict the differences when using the dense, mixed and sparse network configurations. The figure also shows that in all cases the 7-parameter model deteriorates the final $\Delta H_{k l}$ accuracy by approximately $30 \%$, compared to the 3-parameter model. However, it should be noted that all these differences are at the sub-cm level. At this point we can say that the parametric model is not a major contributing factor to the overall achievable accuracy of relative GPS levelling. Going back to Table 2, we can see that relative GPS levelling results in sub-decimeter accuracy for a $10 \mathrm{~km}$ baseline, regardless of the parametric model chosen. The main factor that contributes to the achievable accuracy is the baseline length, as it comes into play through the estimation of the a-priori accuracy of GPS and geoid height data (as previously mentioned in the test description). When the baseline length is increased up to $100 \mathrm{~km}$, the relative GPS levelling accuracy degrades to over $40 \mathrm{~cm}$. By changing the accuracy of the GPS and geoid height determination at the newly established baseline, these results will change accordingly.

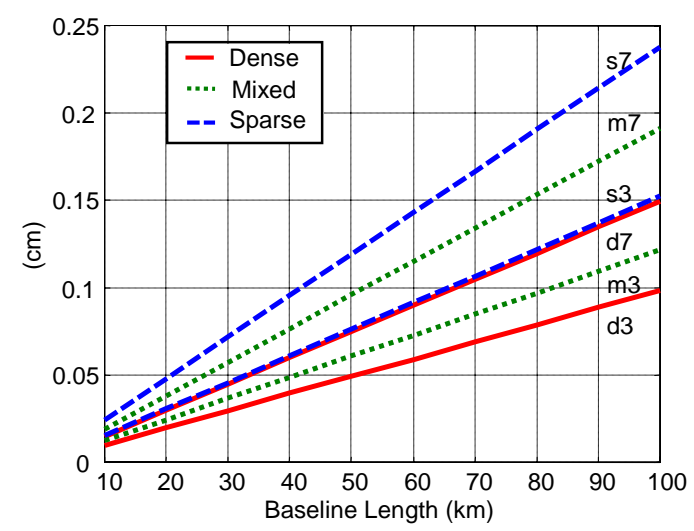

Fig. 4 Accuracy contribution of the corrector surface model to GPS levelling

It is well known that the process of traditional precise spirit levelling suffers from a number of practical limitations, due to the roughness of terrain, harsh environmental conditions, and restricted lineof-sight. In Canada this is most evident when we look at the distribution of vertical control stations, since the northern parts of the country are very 
poorly surveyed. A major advantage of GPS levelling is the fact that GPS observations are not affected (as much) by the practical limitations of spirit levelling. Therefore, we conducted a number of investigations to determine the accuracy on a new baseline established through relative GPS levelling between existing control points in northern Alberta (AB). This area is situated approximately $700 \mathrm{~km}$ to $1000 \mathrm{~km}$ north of the center of the test network, and levelling points are typically more sparsely distributed than in the southern part of the province. For the results that follow, the standard error of the GPS and geoid height differences were held fixed at $4 \mathrm{~cm}$ and $5 \mathrm{~cm}$, respectively, regardless of the baseline length. The results for the achievable accuracy of GPS levelling for baselines situated in northern Alberta (away from the original test network; see Fig. 3) are shown in Table 3.

Table 3. Results for baselines in northern $\mathrm{AB}$

\begin{tabular}{c|c|ccc}
\hline $\begin{array}{c}\text { Dist to orig. } \\
(\mathrm{km})\end{array}$ & $\begin{array}{c}D \\
(\mathrm{~km})\end{array}$ & $\begin{array}{c}\text { 3-param } \\
(\mathrm{cm})\end{array}$ & $\begin{array}{c}\text { 4-param } \\
(\mathrm{cm})\end{array}$ & $\begin{array}{c}\text { 7-param } \\
(\mathrm{cm})\end{array}$ \\
\hline \multicolumn{5}{c}{ Accuracy contribution of the corrector surface model } \\
\hline 730 & 27 & 0.101 & 0.092 & 0.304 \\
760 & 74 & 0.822 & 0.761 & 4.887 \\
890 & 31 & 0.294 & 0.253 & 2.127 \\
\hline \multicolumn{5}{c}{ Accuracy of GPS levelling $\sigma_{\Delta H}$} \\
\hline 730 & 27 & 6.404 & 6.404 & 6.410 \\
760 & 74 & 6.448 & 6.456 & 8.055 \\
890 & 31 & 6.408 & 6.410 & 6.747 \\
\hline
\end{tabular}

It is evident from the above table that for the case where the new baseline is situated farther away from the original test network, the influence of the third term in Eq. (11) becomes more prevalent. For instance, in the case of the 7-parameter model for a $31 \mathrm{~km}$ baseline that was located approximately $890 \mathrm{~km}$ north of the center of the original test network, the value of the third term is over $2 \mathrm{~cm}$. This is significant when a relative orthometric height accuracy of $6.7 \mathrm{~cm}$ is achieved. Another similar example is given by the $74 \mathrm{~km}$ baseline, where the accuracy contribution from the corrector surface parametric model is approximately $4.9 \mathrm{~cm}$.

\section{Conclusions}

A number of different investigations were conducted in order to analyze the effects of (i) the individual height data (GPS, levelling, geoid) accuracy and (ii) the accuracy of the corrector surface parameters, on orthometric height determination via relative GPS levelling. Overall, the influence of the accuracy of the corrector surface parameters was minimal compared to the accuracy contribution of GPS and geoid information. However, if we focus on the accuracy of the corrector surface parameters only, there is a slight difference (at the cm-level) when (i) using different geometrical network configurations, and (ii) using different types of parametric models for the corrector surface. Future studies will involve the use of actual height data values which allows for testing the 'reliability' of the input $\mathrm{CV}$ matrices $C_{\Delta h}, C_{\Delta H}$, and $C_{\Delta N}$ and the form of the parametric model via variance component estimation.

\section{References}

Featherstone WE (2000) Refinement of Gravimetric Geoid Using GPS and Levelling Data. Journal of Surveying Engineering, Vol. 126, Issue 2, pp. 27-56.

Fotopoulos G (2000) Parameterization of DGPS Carrier Phase Errors Over a Regional Network of Reference Stations. MSc. Thesis, University of Calgary, Dept. of Geomatics Engineering, Report No. 20142.

Heiskanen WA and Moritz H (1967) Physical Geodesy. WH Freeman, San Francisco.

Jiang Z and Duquenne, H (1995) Optimal fitting of gravity geoid to GPS levelling points. Latest Developments in the Computation of Regional Geoids, Proceedings of Session G4, EGS XX General Assembly, ed. M. Vermeer, Hamburg, Germany, 3-7 April, 1995, pp. 33-42.

Kearsley AHW, Ahmad Z and Chan A (1993) National Height Datums, Levelling, GPS Heights and Geoids. Aust. J. Geod. Photogram. Surv., No. 59, pp. 53-88.

Kotsakis C and Sideris MG (1999) On the adjustment of combined GPS/levelling/geoid networks. Journal of Geodesy, Vol. 73, No. 8, pp. 412-421.

National Geodetic Survey (1994) Input Formats and Specifications of the National Geodetic Survey Data Base: Volume II, Vertical Control Data. Systems Development Division, National Geodetic Survey (NGS), NOAA, Silver Spring, MD.

Ollikainen M (1997) Determination of Orthometric Heights Using GPS Levelling. Publication of the Finnish Geodetic Institute, No. 123.

van Onselen K (1997) Quality Investigation of Vertical Datum Connection. Delft University of Technology, DEOS Report No. 97.3.

Véronneau M (1997) The GSD95 geoid model for Canada. Proceedings of the IAG International Symposium on Gravity, Geoid and Marine Geodesy, Sept. 30 - Oct. 5, Tokyo, 1996, Springer-Verlag, Vol. 117, pp. 573-580. 